\title{
Toward Metacognitive Radars: Concept and Applications
}

\author{
Kumar Vijay Mishra ${ }^{\dagger \ddagger}$, Bhavani Shankar M. R. and Björn Ottersten $^{\ddagger}$ \\ ${ }^{\dagger}$ United States CCDC Army Research Laboratory, Adelphi, MD 20783 USA \\ ${ }^{\ddagger}$ Interdisciplinary Centre for Security, Reliability and Trust (SnT), University of Luxembourg 1855, Luxembourg \\ Email: \{kumar.mishra@ext., bhavani.shankar@, bjorn.ottersten@\}uni.lu
}

\begin{abstract}
We introduce a metacognitive approach to optimize the radar performance for a dynamic wireless channel. Similar to the origin of the cognitive radar in the neurobiological concept of cognition, metacognition also originates from neurobiological research on problemsolving and learning. Broadly defined as the process of learning to learn, metacognition improves the application of knowledge in domains beyond the immediate context in which it was learned. We describe basic features of a metacognitive radar and then illustrate its application with some examples such as antenna selection and resource sharing between radar and communications. Unlike previous works in communications that only focus on combining several existing algorithms to form a metacognitive radio, we also show the transfer of knowledge in a metacognitive radar. A metacognitive radar improves performance over individual cognitive radar algorithms, especially when both the channel and transmit/receive hardware are changed.

Index Terms-Cognitive radar, cognitive radio, deep learning, metacog-
\end{abstract} nitive radar, resource sharing, transfer learning.

\section{INTRODUCTION}

In recent years, cognitive radars have captured significant attention. The key strength of such a system is its ability to learn the channel or target environment and then adapt both the transmitter and receiver to provide an enhanced performance [1, 2]. On the contrary, a conventional radar optimizes only receive processing in response to changes in the target environment. During the past two decades, cognition in radar has matured from a conceptual stage [3-5] to the implementation in hardware [6-8]. Several diverse applications of cognitive radar have been suggested such as spectrum sharing [9], adaptable beampattern design [10], enhanced tracking [11, 12], and resource allocation [13].

In general, a cognitive radar is designed to apply a single specific framework or algorithm to achieve its desired performance based on a pre-determined criteria. Since radars perform a variety of tasks such as detection, estimation and tracking, in practice, a single cognitive radar framework is insufficient to address changes in the system hardware and channel environment over long periods of time. For example, a radar that cognitively selects the beam direction to avoid jamming [14] may need a different cognitive strategy when jammer is co-located with the target. Further, as the complexity of radar system increases (e.g. use of multiple antennas and waveforms) and the channel conditions worsen (low signal-to-noise-ratio/SNR and presence of clutter), a single cognitive algorithm is unable to address the changing performance requirements. In such cases, a strategy to combine various cognitive frameworks in a metacognitive radar is highly desirable. In this paper, we focus on the concept of metacognitive radar.

In its original concept, the cognitive radar drew upon the definition of cognition from neurobiology, wherein it is a process through which humans and animals sense and interact with their environment [1]. Similarly, metacognition is a well-studied concept in both neurobiology and educational psychology, often associated with the definition

K. V. M. acknowledges support from the National Academies of Sciences, Engineering, and Medicine via Army Research Laboratory Harry Diamond Distinguished Postdoctoral Fellowship. proposed by John Flavell [15]. It is formally defined as a higher order thinking which actively controls the cognitive processes engaged in learning [16]. This definition often summarizes metacognition as learning about learning or knowing about knowing [17, 18]. At the heart of metacognitive system lie four components: acquiring knowledge about the environment; monitoring different cognition methods; a strategy to use the information obtained; and transfer the learned strategy to a new environment [16].

In wireless communications, some recent works have discussed applications of metacognition. In [19], a metacognitive radio was proposed in the context of efficient spectrum utilization by a cognitive communications system that constantly monitor and acquire the channel state information (CSI). Then, an appropriate cognitive method is selected from a suite of strategies such as genetic algorithms, reinforcement learning, or artificial neural networks. An example of this strategy is precoder-decoder design for interference management which coordinates co-existing multiple transmitters such that their mutual interference aligns at the receivers and occupies only a portion of the signal space. In this context CSI is routinely sensed and estimated. Later, in [20], this concept of metacognitive radio was expanded to a general metacognitive engine which included addressing multiple applications. However, these works only incorporate the first three components of metacognition: acquisition, monitoring and strategy selection. The transfer of learned knowledge to new scenarios, which is essential to demonstrate metacognition, was ignored in these studies.

So far, metacognition in radar remains relatively unexamined. In this work, we introduce the concept of metacognitive radar and explain its key features. Unlike the aforementioned previous works on metacognition in wireless communications, we include all four components of metacognition in our radar formulation. We illustrate this concept through some examples of resource selection and sharing. In our proposed configuration, learning-based methods are critical in enabling metacognition. In fact, use of techniques such as deep learning to empower cognition is synonymous with the original definition of metacognition as learning about learning. Another key technique in this context is application of control- and gametheoretic methods which are capable to model decision making in an environment of conflict and cooperation between rational players [21]. For example, in [21], various games are modeled depending on the information available at the radar and jammer about each other. A metacognitive radar could model various performance objectives as different games and then select the most appropriate one for the situation. A metacognitive radar holds the promise of making the cognitive radars more realistic and efficient by expanding their original sensing cycles.

In the following section, we explain the cognitive radar cycle, introduce its metacognitive counterpart, and provide various illustrative applications. We follow this by an example of resource selection in Section III and validate our method in Section IV. We conclude in Section V. 


\section{Introducing a Metacognitive Radar}

A conventional cognitive radar [22] may be viewed as a dynamic closed-loop system employing three steps (Fig. 1). In sense or observe stage, the radar gathers all the information from the target environment. It then decides or learns by applying some degree of intelligence which includes learning, planning and decision making methods. Finally, the radar adapts to the change in the target channel by reconfiguring the transmitter and receiver in order to be as flexible as possible to enhance the performance. This constitutes a typical cognitive cycle and is also a common feature in cognitive radio systems. All three steps are performed cyclically. In a two step model of cognitive radar, the sense and decide stages are grouped under perception and adapt step as an action.

\section{A. Metacognitive Cycle}

It is pertinent to remark the importance of metacognition as separate from plain cognition. Since the cognitive cycle is a closedloop system, there is no provision of altering any of the steps once the cycle has kicked in a operational system. This leads to an inherent inflexibility of the system to adapt to drastic change in the channel conditions, change of engineering modules, or the operating objective or all of these. Hence, the radar must include multiple strategies with their own cognitive cycles. The selection of the appropriate strategy is handled by metacognition.

Motivated by its psychological definitions [16], we define a metacognitive cycle with four key steps 1 . The knowledge acquisition step collates the assumptions inherent to all individual cognitive cycles to generate their decisions. For example, in radarcommunications spectrum sharing scenario, the three cycles may each be tailored to the three different waveforms which only the metacognitive knowledge keeps a record of. In the monitoring step, the metacognitive radar evaluates the strengths and drawbacks of choosing a particular cycle. For example, choosing a reinforcement learning for spectrum sharing would require devising a policy and reward while a deep learning strategy would operate only with a large training set.

The monitoring step is viewed as information flowing from the cognitive cycle to the metacognitive cycle [23]. The reverse of this flow is the strategy/control stage of metacognition, wherein the radar applies a learning tool to select a specific cognitive cycle. For example, a deep learning engine may decide choosing between various strategies for spectrum sharing. As discussed in [20], the addition of this secondary, metacognitive cycle permits control and independent judgment of primary cognition behavior. Metacognition increases the confidence of the radar in its cognitive judgements. When the radar is deployed in slightly different environments that none of the cognitive cycles were prepared for, metacognition imparts the ability to apply knowledge accumulated in the first three steps to the new domain. This final stage of knowledge transfer completes the metacognitive cycle.

\section{B. Applications}

We now elaborate on some sample applications of a metacognitive radar. Our first application of spectrum sharing follows from prior works on metacognitive radio [19]. The second example is concerned with the allocation of resources such as power in a radar-communications scenario. The third example of metacognitive antenna selection follows from our previous works [13, 24, 25].

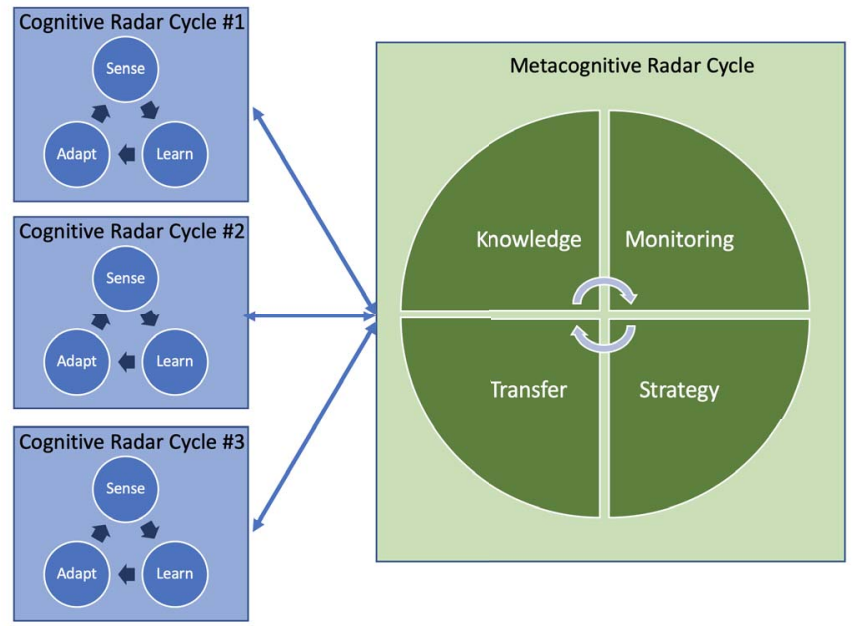

Fig. 1. Metacognitive radar cycle consists of knowledge acquisition, monitoring, strategy or control and transfer stages. A metacognitive engine monitors various cognitive strategies employed by the radar. Each of the cognitive strategy has its conventional sense-learn-adapt cycle with perception and action stages.

1) Metacognitive spectrum sharing: As seen in metacognitive radio applications, the concept of metacognition is very useful for addressing spectrum sharing problem. Spectrum scarcity is a major current concern for the radio community and, in particular, for both radar and communications applications [26, 27]. As a consequence, spectrum sharing strategies have recently gained considerable attention $[28,29]$. Current standardization effort in wireless communications for finding new spectrum opportunities aims at using bands that are not dedicated to communications for communications use. The European Licence Shared Access (LSA) effort [30] aims at allowing a licensee, to use the spectrum of an incumbent who has a spectrum access right, following certain rules. The idea is to enable mobile telephony ( $4 \mathrm{G}$ and $5 \mathrm{G})$ operators to use this spectrum in areas apart from the airport radio range. The LSA relies for that on the use of geo-location data bases (GLDB) that are consulted before the connection of the LSA users. Licensed Shared Access is a big step forward to save cost once implemented in the operators network and solve spectrum scarcity in the lower bands below $6 \mathrm{GHz}$. On the other hand, the US Citizen Band Radio System (CBRS) [26] initiative has been launched as a way to compensate US delay compared to the LSA initiative. Then in order to bring some added value compared to LSA, US standardization effort consisted in adding an extended version of LSA to be used in the $3.5 \mathrm{GHz}$ band $(3550-3700 \mathrm{MHz})$.

A metacognitive spectrum sharing radar in such a situation should operate as follows. There are various learning and non-learning cognitive approaches to spectrum sharing depending on the application e.g. reinforcement learning at cognitive spectrum sharing for autonomnous driving [31] and Xampling for surveillance cognitive radar-radio applications [9]. A metacognitive engine could choose between the two methods depending on the deployment of the radar in those situations. However, when the system is deployed in an application that none of the two techniques are developed for, the metacognitive radar exploits its knowledge and adapts the system to the new application.

2) Metacognitive power allocation: Similar to spectrum sharing, power allocation between a radar and communications systems can also be modeled as a metacognitive radar problem [32]. For instance, 
let the radar and communications transmit power be $P_{R}$ and $P_{C}$, respectively. Define the complex-Gaussian-distributed gains for the various discrete-time channel impulse responses with zero mean and variances $\sigma_{t}^{2}, \sigma_{i}^{2}, \sigma_{c}^{2}, \sigma_{f}^{2}$, and $\sigma_{r}^{2}$, respectively, as follows: $h_{T} \sim \mathcal{C N}\left(0, \sigma_{t}^{2}\right)$ : radar transmitter to the target and back to the radar receiver; $h_{I} \sim \mathcal{C N}\left(0, \sigma_{i}^{2}\right)$ : radar transmitter to clutter and back to the radar receiver; $h_{C} \sim \mathcal{C N}\left(0, \sigma_{c}^{2}\right)$ : radar transmitter to the communications receiver; $h_{F} \sim \mathcal{C N}\left(0, \sigma_{f}^{2}\right)$ : communications transmitter to the communications receiver; $h_{R} \sim \mathcal{C N}\left(0, \sigma_{r}^{2}\right)$ : radar transmitter to the target and clutter and then to the communications receiver; and $w[n] \sim \mathcal{C N}\left(0, \sigma_{w}^{2}\right)$ and $v[n] \sim \mathcal{C N}\left(0, \sigma_{v}^{2}\right)$ denote the noise trails at the radar and communications receivers, respectively.

At the radar receiver, the signal-to-interference-plus-clutter-plusnoise-ratio (SICNR) is

$$
\operatorname{SICNR}_{R}=\frac{\sigma_{t}^{2} P_{R}}{\sigma_{c}^{2} P_{C}+\sigma_{i}^{2} P_{R}+\sigma_{w}^{2}}
$$

The radar may also have a maximum power, maximum interference and minimum SICNR constraints so that

$$
\begin{gathered}
0 \leq P_{R} \leq P_{R, \text { max }}, \\
\sigma_{c}^{2} P_{C} \leq T_{C, \text { max }}, \\
\text { and } \mathrm{SICNR}_{R} \geq \operatorname{SICNR}_{R, \text { min }},
\end{gathered}
$$

where $P_{R, \max }, T_{C, \max }$, and $\mathrm{SICNR}_{R, \min }$ are pre-defined constants.

At the communications receiver, the signal-to-interference-plusnoise-ratio (SINR) is

$$
\operatorname{SINR}_{C}=\frac{\sigma_{f}^{2} P_{C}}{\sigma_{r}^{2} P_{R}+\sigma_{v}^{2}}
$$

The communications receiver may have the maximum power, maximum interference and minimum SINR constraints as

$$
\begin{gathered}
0 \leq P_{C} \leq P_{C, \max }, \\
\sigma_{r}^{2} P_{R} \leq T_{R, \max }, \\
\text { and } \operatorname{SINR}_{C} \geq \mathrm{SINR}_{C, \min },
\end{gathered}
$$

where $P_{C, \max }, T_{R, \max }$, and $\mathrm{SINR}_{C, \min }$ are pre-defined constants.

Let the game be the triplet $\mathcal{G}=\langle\mathcal{K}, \mathcal{S}, \mathcal{U}\rangle$ where $\mathcal{K}$ is the set of players with cardinality $|\mathcal{K}|=K, \mathcal{S}=S_{1} \times \cdots \times S_{K}$ is the space comprising of strategies $\left\{S_{i}\right\}_{i=1}^{K}$ of all players, and $\mathcal{U}=\left\{u_{1}, \cdots, u_{K}\right\}$ is the set of utility functions of each player which map their strategies to a real line, i.e., $u_{i}: S_{i} \rightarrow \mathbb{R}, i=1, \cdots, K$. In our spectral coexistence problem, $|\mathcal{K}|=2$ and $K=1$ and 2 corresponds to radar and communications, respectively. Further $S_{1}=\left[0, P_{R, \max }\right]$ and $S_{2}=\left[0, P_{C, \max }\right]$.

The utility functions are given by the difference of payoff (maximization of SICNR) and the cost functions (minimization of power). As an example, the respective utility functions could be

$$
u_{1}=\ln \left(\operatorname{SICNR}_{R}-\operatorname{SICNR}_{R, \text { min }}\right)-\left(\mu_{1} \sigma_{t}^{2} P_{R}+\gamma_{1} \sigma_{r}^{2} P_{R}\right)
$$

$$
\text { and } u_{2}=\ln \left(\operatorname{SINR}_{C}-\operatorname{SINR}_{C, \min }\right)-\left(\mu_{2} \sigma_{f}^{2} P_{C}+\gamma_{2} \sigma_{c}^{2} P_{C}\right) \text {, }
$$

where $\mu_{i}$ and $\gamma_{i}, i=1,2$ are to be determined. The power allocation is determined by solving for the values of these utility functions. The metacognitive system models the interaction between the radar and communications as a cooperative or non-cooperative game depending on the information available and solves the resulting optimization problem for the desired resource allocation.
3) Metacognitive antenna selection: Sparse array selection is one of the common tasks performed by cognitive radars [13]. Larger arrays have high associated cost, area and computational load. To address this problem, a cognitive radar deploys a full array and then select an optimal (sparse) subarray to transmit and receive the signals in response to changes in the target environment. This task is achieved through a variety of techniques such as optimization, greedy search, random array selection and deep learning [13, 33].

Briefly, the cognitive cycle in this problem operates as follows. For a fixed array geometry, assume a phased array employed by a radar that performs angle estimation using sparse recovery methods. During the very first scan, full array is active and the received signal from this scan is fed to the network. The cognitive radar goal is to find an optimal antenna array for the next few scans in which fewer antennas than the full array will be used. For deep learning, the radar employs a trained network that chooses an optimal array using the covariance of the received signal. The optimal array provides the lowest estimation error in the direction-of-arrival. The same criterion is used by the optimization and greedy search methods For random array selection, no such criterion is used. All four methods differ in their computation speeds. Thus, a metacognitive radar chooses between these different strategies depending on the available computing resources. When the array geometry is changed, a metacognitive radar will apply a technique for knowledge transfer. In the following section, we explain this last stage with a concrete example. For all other stages, we refer the reader to our prior works $[13,33]$. The example is based on our recent work on sparse array selection for arbitrary geometries [25].

\section{Example: Antenna Selection Across Geometries}

Consider an $M$-element antenna array receiving a signal $s\left(t_{i}\right)$ from the direction $\Theta=(\theta, \phi)$ where $\theta$ and $\phi$ are the elevation and the azimuth angles of the source with respect to the antenna array. We assume that the received signal is narrowband and the source is in the far-field of the antenna array. Then, the output of the antenna array is given by

$$
\mathbf{y}\left(t_{i}\right)=\mathbf{a}(\Theta) s\left(t_{i}\right)+\mathbf{n}\left(t_{i}\right), \quad 1 \leq i \leq T,
$$

where $T$ is the number of snapshots, $\mathbf{y}\left(t_{i}\right)=\left[y_{1}\left(t_{i}\right), \ldots, y_{M}\left(t_{i}\right)\right]^{T}$ and $y_{m}\left(t_{i}\right)$ denotes the output of the $m$ th antenna for the $i$ th snapshot. $\mathbf{n}\left(t_{i}\right)=\left[n_{1}\left(t_{i}\right), \ldots, n_{M}\left(t_{i}\right)\right]^{T}$ is the noise vector and $n_{m}\left(t_{i}\right)$ is additive white Gaussian noise (AWGN) with variance $\sigma_{n}^{2} \cdot \mathbf{a}(\Theta)=\left[a_{1}(\Theta), \ldots, a_{M}(\Theta)\right]^{T}$ is the steering vector and $a_{m}(\Theta)=\exp \left\{-j \frac{2 \pi}{\lambda} \mathbf{p}_{m}^{T} \mathbf{r}(\Theta)\right\}$ where $\mathbf{p}_{m}=\left[x_{m}, y_{m}, z_{m}\right]^{T}$ is the position of the $m$ th antenna in a Cartesian coordinate system, $\mathbf{r}(\Theta)=$ $[\cos (\phi) \sin (\theta), \sin (\phi) \sin (\theta), \cos (\theta)]^{T}$ and $\lambda$ is the wavelength of the baseband signal.

\section{A. Cognitive Cycle}

In sparse array selection problem we consider an $M$-element antenna array where $K$ out of $M$ are to be selected in the sense that the selected subarray provides the "best" DOA estimation performance. Then we have $C=\left(\begin{array}{c}M \\ K\end{array}\right)$ possible subarray choices. Hence it can be considered as a classification problem with $C$ classes We formulate the problem statement as: predicting the class which corresponds to the "best" subarray when the array output is given.

We consider the antenna selection problem in deep learning context [13] and design a deep CNN to classify the input data of the network (the antenna array output) to select the "best" subarray for DOA estimation. We transfer the knowledge in the training data to target domain for antenna selection with another array geometry. In the sequel, we first discuss the generation of the training data. 


\section{B. Knowledge Transfer Across Different Array Geometries}

We treat the antenna selection problem as classification problem with $C$ classes (i.e., $C$ many subarray configurations). Each class is labeled with the positions of the antennas of the subarray corresponding to that class. Let $P_{k}^{c}=\left\{p_{x_{k}}^{c}, p_{y_{k}}^{c}, p_{z_{k}}^{c}\right\}$ be the set of antenna coordinates in the $c$ th subarray for $k=1, \ldots, K$. The positions of the antennas in the $c$ th subarray constitute the set $\mathcal{S}_{c}=\left\{P_{1}^{c}, P_{2}^{c}, \ldots, P_{K}^{c}\right\}$, and the set of all classes are given as $\mathcal{S}=\left\{\mathcal{S}_{1}, \mathcal{S}_{2}, \ldots, \mathcal{S}_{C}\right\}$, which contains all the subarray configurations. In order to select the best subarrays in $\mathcal{S}$, we compute the CRB $\forall \mathcal{S}_{c}$. We define the absolute CRB for the direction $\Theta$ and $\mathcal{S}_{c}$ as

$$
\kappa\left(\Theta, \mathcal{S}_{c}\right)=1 / 2\left(\kappa\left(\theta, \mathcal{S}_{c}\right)^{2}+\kappa\left(\phi, \mathcal{S}_{c}\right)^{2}\right)^{2},
$$

where $\kappa\left(\theta, \mathcal{S}_{c}\right)$ and $\kappa\left(\phi, \mathcal{S}_{c}\right)$ are the CRB terms for elevation and azimuth angles respectively $[13,34]$. The CRB for $\theta$ in a single source scenario is computed as follows [34]

$$
\kappa\left(\theta, \mathcal{S}_{c}\right)=\frac{\sigma_{n}^{2}}{2 T \operatorname{Re}\left\{\left(\dot{\mathbf{a}}_{c_{\theta}}^{H}\left[\mathbf{I}_{K}-\mathbf{a}_{c} \mathbf{a}_{c}^{H} / K\right] \dot{\mathbf{a}}_{c_{\phi}}\right) \odot\left(\sigma_{s}^{4} \mathbf{a}_{c}^{H} \mathbf{R}_{c}^{-1} \mathbf{a}_{c}\right)\right\}},
$$

and for the azimuth angle, $\kappa\left(\phi, \mathcal{S}_{c}\right)$ as

$$
\kappa\left(\phi, \mathcal{S}_{c}\right)=\frac{\sigma_{n}^{2}}{2 T \operatorname{Re}\left\{\left(\dot{\mathbf{a}}_{c_{\phi}}^{H}\left[\mathbf{I}_{K}-\mathbf{a}_{c} \mathbf{a}_{c}^{H} / K\right] \dot{\mathbf{a}}_{c_{\theta}}\right) \odot\left(\sigma_{s}^{4} \mathbf{a}_{c}^{H} \mathbf{R}_{c}^{-1} \mathbf{a}_{c}\right)\right\}},
$$

where $\odot$ is the dot product and $\mathbf{a}_{c} \in \mathbb{C}^{K}$ denotes the steering vector corresponding to the subarray with positions $\mathcal{S}_{c} . \mathbf{R}_{c}=$ $1 / T \sum_{i=1}^{T} \mathbf{y}_{c}\left(t_{i}\right) \mathbf{y}_{c}^{H}\left(t_{i}\right)$ is the sample covariance matrix for the subarray output $\mathbf{y}_{c}\left(t_{i}\right)=\mathbf{a}_{c} s\left(t_{i}\right)+\mathbf{n}_{c}\left(t_{i}\right)$ and $\mathbf{I}_{K}$ is a $K \times K$ unit matrix. $\dot{\mathbf{a}}_{c_{\theta}}=\frac{\partial \mathbf{a}_{c}}{\partial \theta}$ and $\dot{\mathbf{a}}_{c_{\phi}}=\frac{\partial \mathbf{a}_{c}}{\partial_{\phi}}$ are the partial derivatives of $\mathbf{a}_{c}$ with respect to $\theta$ and $\phi$ respectively. $\sigma_{s}^{2}$ and $\sigma_{n}^{2}$ are the signal and noise variances. For simplicity, we select $\sigma_{s}^{2}=1$ and define the signal to noise ratio in the training data as $\operatorname{SNR}_{\text {TRAIN }}=10 \log _{10}\left(\sigma_{s}^{2} / \sigma_{n}^{2}\right)$.

Among $C$ subarray configurations, we found that the number of subarrays that provide the "best" DOA estimation performance (i.e., the lowest $\mathrm{CRB}$ ) is much less than $C$. Hence we labeled them in the set $\mathcal{B}=\left\{b_{1}, b_{2}, \ldots, b_{\bar{C}}\right\}$ where

$$
b_{\bar{c}}=\arg \min _{c=1, \ldots, C} \kappa\left(\Theta, \mathcal{S}_{c}\right),
$$

which implies that there are $\bar{C}$ subarrays among $C$ that provides the lowest CRB. This is due to the configuration of the array structure [13].

The training dataset for the $\mathrm{CNN}$ structure is $\mathcal{D}=(\mathbf{X}, z)$ where $z \in \mathcal{B}$ denotes the label that is the best subarray index. $\mathbf{X}=\left\{\mathbf{X}_{h}\right\}_{h=1}^{3}$ is the input which is a three channel data. The first channel, $\mathbf{X}_{1} \in$ $\mathbb{R}^{M \times M}$, contains the angle information of the array covariance matrix as $\left[\mathbf{X}_{1}\right]_{i, j}=\angle\left\{[\mathbf{R}]_{i, j}\right\}$ for the $(i, j)$ th entry. The second and the third channels are the real and imaginary parts of the covariance matrix respectively. In particular, $\left[\mathbf{X}_{2}\right]_{i, j}=\mathbb{R e}\left\{[\mathbf{R}]_{i, j}\right\}$ and $\left[\mathbf{X}_{3}\right]_{i, j}=$ $\operatorname{Im}\left\{[\mathbf{R}]_{i, j}\right\}$.

We design a deep CNN structure which is composed of 14 layers. The first layer is the input layer which accepts the input of size $M \times M \times 3$. The $\{2,4,6,8\}$ th layers are convolutional layers each of which has 64 filter with the size of $2 \times 2$. In the 10th and 12 th layers, there are fully connected layers with 1024 units whose $50 \%$ is randomly selected to avoid overfitting. After each convolutional and fully connected layers (in the $\{3,5,7,9,11,13\}$ th layers), there is a rectified linear unit $(\operatorname{ReLU})$ layer where $\operatorname{ReLU}(x)=\max (x, 0)$. In the last layer, there is a classification layer with $\bar{C}$ units where a softmax function is used to obtain the probability distribution of the classes. In order to train the network, the training data is collected

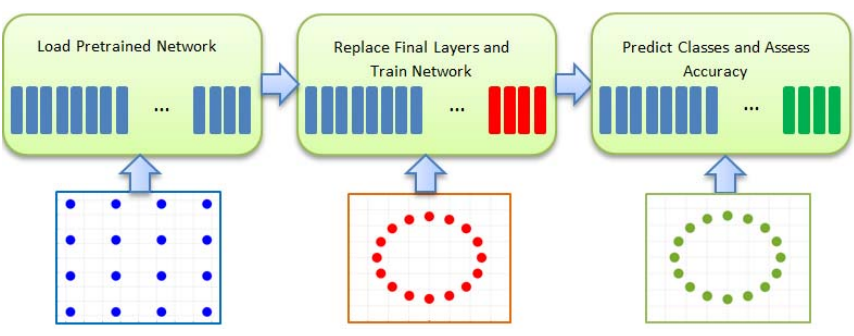

Fig. 2. Transfer learning framework for knowledge transfer across URA and UCA geometries.

for $P$ directions and $L$ realizations. Then, the network is realized in MATLAB on a PC with 768-core GPU. Stochastic gradient decent algorithm is used to update the network parameters with the learning rate 0.01 and mini-batch size 500 for 50 epochs.

Once the training data is in the source domain, $\mathcal{D}_{\mathrm{S}}$, is generated with the source array geometry. Then it is used to train the network and we obtain $\mathrm{CNN}_{\mathrm{S}}$ where the subscript stands for the source domain. The pretrained network (i.e., $\mathrm{CNN}_{\mathrm{S}}$ ) is modified by replacing the classification layer with the one that is appropriate with target data labels. The weights in all of the convolutional layers are kept fixed to preserve the learned features in the source domain. We call the resulting network $\mathrm{CNN}_{\mathrm{TR}}$ which is trained with the target training set, $\mathcal{D}_{\mathrm{T}}$, generated with the target array geometry.

\section{NumericAl Simulations}

We first present the performance of the proposed CNN approach for the source domain case where different array geometries such as URA and UCA are considered with different settings. We collected data for $P_{\text {TRAIN }}=100$ equally spaced directions in the interval $\left[0^{\circ}, 359^{\circ}\right]$ azimuth plane and for $L_{\text {TRAIN }}=100$ realizations. $\operatorname{SNR}_{\text {TRAIN }}=20 \mathrm{~dB}$ and $T_{\text {TRAIN }}=100$ is selected. The network is tested for different $\mathrm{SNR}_{\mathrm{TEST}}$ levels for $J_{T}=100$ Monte Carlo trials. We obtain above $90 \%$ validation accuracy for the training data in all cases as in [13]. This means that the proposed CNN structure accurately selects the antenna subarray in the "best" sense. In order to present the DOA estimation performance of the selected subarray, the MUSIC algorithm [35] is employed. In this case, we prepared a test data which is separately generated from the training data with different DOA angles which are selected uniform randomly. In Fig. 3, the DOA estimation performance for different arrays are given and compared with the "best" subarray performance which refers to the subarray that provides the lowest CRB value. As it is seen from the figure, our $\mathrm{CNN}$ approach performs well and it closely follows the best subarray performance.

To evaluate the performance of our CNN approach for transfer learning, target domain data is generated for UCA geometry with $M=16, K=6$ and element spacing is $\lambda / 2$. We consider $1 \mathrm{D}$ scenario where the azimuth space is sampled with $P_{T}=10$ directions and $L_{T}=10$ for the target dataset. The source domain data is generated for URA geometry with $M=16, K=6$ with $\lambda / 2$ element spacing. The transfer learning performance is investigated as the size of the source dataset varies. In Fig. $4, P_{S}$, the number of directions in the source dataset, varies as the other parameters are kept fixed as $L_{S}=100$ and $\mathrm{SNR}_{\mathrm{TRAIN}}=15 \mathrm{~dB}$. For each $P_{S}, \mathrm{CNN}_{\mathrm{TR}}$ is generated and trained with target dataset. As seen from Fig. $4, \mathrm{CNN}_{\mathrm{TR}}$ performs much better than $\mathrm{CNN}_{\mathrm{T}}$ even if they are trained with the same data (i.e., target data). The performance of $\mathrm{CNN}_{\mathrm{TR}}$ is attributed to the use of features from the source domain which are not available in target 


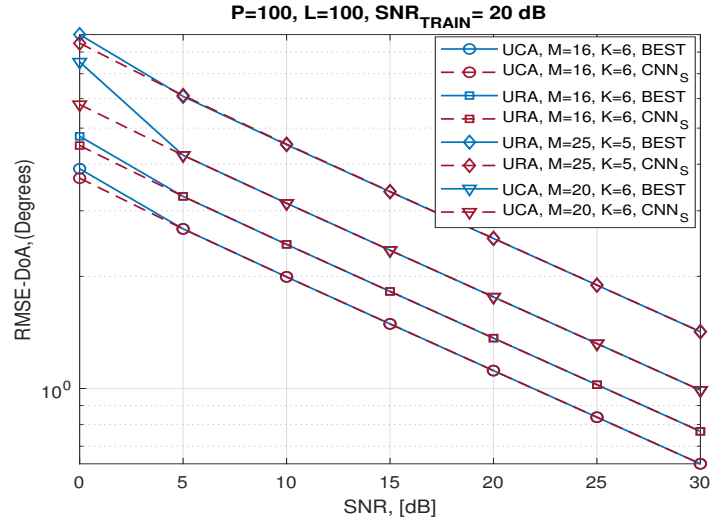

Fig. 3. DOA estimation performance for the $\mathrm{CNN}_{\mathrm{S}}$ for different array settings [25].

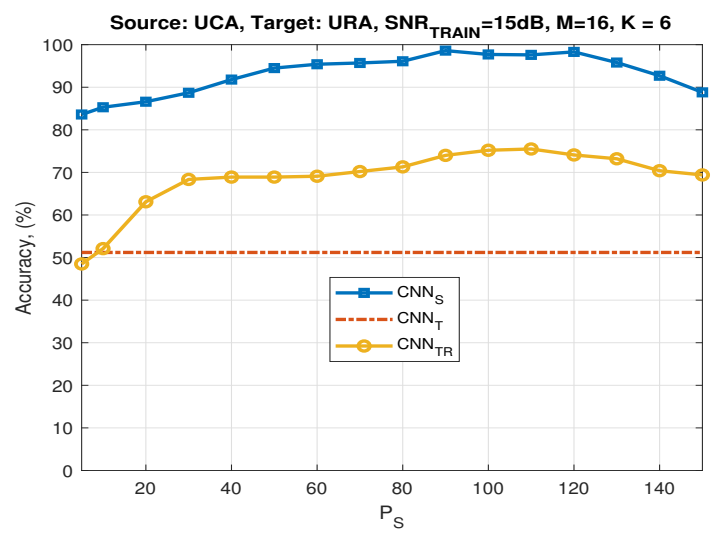

Fig. 4. Performance of $\mathrm{CNN}_{\mathrm{S}}, \mathrm{CNN}_{\mathrm{T}}$ and $\mathrm{CNN}_{\mathrm{TR}}$ vs $P_{S} . L_{S}=100$ and $\mathrm{SNR}_{\mathrm{TRAIN}}=15 \mathrm{~dB}[25]$.

domain. We obtain approximately $20 \%$ increase in the classification accuracy. We observe that transfer learning works if the source dataset is large so that more features can be transferred to target domain. While the accuracy of $\mathrm{CNN}_{\mathrm{S}}$ is similar for different $P_{S}$ values, it significantly affects the performance of $\mathrm{CNN}_{\mathrm{TR}}$. From Fig.4, we see that $P_{S}=100$ is a good choice for transfer learning where the source data is 100 times larger than target data. The performance of $\mathrm{CNN}_{\mathrm{TR}}$ decreases after a certain $P_{S}$ value since collecting more dense direction data leads to ambiguities in labeling.

\section{SUMmary}

We introduced the concept of metacognitive radar whose aim is to impart additional confidence to the decisions of a cognitive radar. This is achieved by collating several cognitive cycles in the system for various scenarios and selecting the most appropriate one for a given situation. We discussed key elements of a metacognitive radar and its possible applications. Finally, we illustrated a cognitive radar antenna selection by including the knowledge transfer step.

\section{REFERENCES}

[1] S. Haykin, "Cognitive radar: A way of the future," IEEE Signal Processing Magazine, vol. 23, no. 1, pp. 30-40, 2006.

[2] J. R. Guerci, Cognitive radar: The knowledge-aided fully adaptive approach. Artech House, 2010.

[3] K. V. Mishra and Y. C. Eldar, "Performance of time delay estimation in a cognitive radar," in IEEE International Conference on Acoustics, Speech and Signal Processing, 2017, pp. 3141-3145.
[4] — "Sub-Nyquist radar: Principles and prototypes," in Compressed Sensing in Radar Signal Processing, A. D. Maio, Y. C. Eldar, and A. Haimovich, Eds. Cambridge University Press, 2019, pp. 1-48.

[5] A. F. Martone, K. I. Ranney, K. Sherbondy, K. A. Gallagher, and S. D. Blunt, "Spectrum allocation for noncooperative radar coexistence," IEEE Transactions on Aerospace and Electronic Systems, vol. 54, no. 1, pp. 90-105, 2017.

[6] Z. Slavik and K. V. Mishra, "Cognitive interference mitigation in automotive radars," in IEEE Radar Conference, 2019, in press.

[7] J. W. Owen, B. Ravenscroft, B. H. Kirk, S. D. Blunt, C. T. Allen, A. F. Martone, K. D. Sherbondy, and R. M. Narayanan, "Experimental demonstration of cognitive spectrum sensing \& notching for radar," in IEEE Radar Conference, 2018, pp. 0957-0962.

[8] B. Ravenscroft, J. W. Owen, J. Jakabosky, S. D. Blunt, A. F. Martone, and K. D. Sherbondy, "Experimental demonstration and analysis of cognitive spectrum sensing and notching for radar," IET Radar, Sonar \& Navigation, vol. 12, no. 12, pp. 1466-1475, 2018.

[9] D. Cohen, K. V. Mishra, and Y. C. Eldar, "Spectrum sharing radar: Coexistence via Xampling," IEEE Transactions on Aerospace Electronic Systems, vol. 29, pp. 1279-1296, 32018.

[10] M. Alaee-Kerahroodi, K. V. Mishra, M. R. B. Shankar, and B. Ottersten, "Discrete phase sequence design for coexistence of MIMO radar and MIMO communications," in IEEE International Workshop on Signal Processing Advances in Wireless Communications, 2019, pp. 1-5.

[11] N. Sharaga, J. Tabrikian, and H. Messer, "Optimal cognitive beamforming for target tracking in MIMO radar/sonar," IEEE Journal of Selected Topics in Signal Processing, vol. 9, no. 8, pp. 1440-1450, 2015.

[12] K. L. Bell, C. J. Baker, G. E. Smith, J. T. Johnson, and M. Rangaswamy, "Cognitive radar framework for target detection and tracking," IEEE Journal of Selected Topics in Signal Processing, vol. 9, no. 8, pp. 14271439, 2015.

[13] A. M. Elbir, K. V. Mishra, and Y. C. Eldar, "Cognitive radar antenna selection via deep learning," IET Radar, Sonar \& Navigation, vol. 13, no. 6, pp. 871-880, 2019.

[14] X. Jiang, F. Zhou, Y. Jian, and H. Xi, "An optimal POMDP-based antijamming policy for cognitive radar," in IEEE Conference on Automation Science and Engineering, 2017, pp. 938-943.

[15] J. H. Flavell, "Metacognition and cognitive monitoring: A new area of cognitive-developmental inquiry." American psychologist, vol. 34 no. 10 , p. $906,1979$.

[16] J. A. Livingston, "Metacognition: An overview," Educational Resources Information Center, U. S. Department of Education, Tech. Rep. TM034808, 2003.

[17] J. Metcalfe and A. P. Shimamura, Metacognition: Knowing about knowing. MIT press, 1994

[18] J. Dunlosky and J. Metcalfe, Metacognition. Sage Publications, 2008.

[19] H. Asadi, H. Volos, M. M. Marefat, and T. Bose, "Metacognitive radio engine design and standardization," IEEE Journal on Selected Areas in Communications, vol. 33, no. 4, pp. 711-724, 2015.

[20] _ "Metacognition and the next generation of cognitive radio engines," IEEE Communications Magazine, vol. 54, no. 1, pp. 76-82, 2016.

[21] X. Song, P. Willett, S. Zhou, and P. B. Luh, "The MIMO radar and jammer games," IEEE Transactions on Signal Processing, vol. 60, no. 2, pp. 687-699, 2012.

[22] J. Mitola, "Cognitive radio: An integrated agent architecture for software defined radio," Ph.D. dissertation, Royal Institute of Technology, Sweden, 2000.

[23] A. Achtziger, S. E. Martiny, G. Oettingen, and P. M. Gollwitzer, "Metacognitive processes in the self-regulation of goal pursuit," Social metacognition, pp. 121-139, 2012.

[24] A. M. Elbir and K. V. Mishra, "Joint antenna selection and hybrid beamformer design using unquantized and quantized deep learning networks," arXiv e-prints, 2019.

[25] _ _ "Sparse array selection across arbitrary sensor geometries with deep transfer learning," 2020, under review.

[26] K. V. Mishra, M. R. Bhavani Shankar, V. Koivunen, B. Ottersten, and S. A. Vorobyov, "Toward millimeter wave joint radar communications: A signal processing perspective," IEEE Signal Processing Magazine, vol. 36, pp. 100-114, 2019.

[27] A. Ayyar and K. V. Mishra, "Robust communications-centric coexistence for turbo-coded OFDM with non-traditional radar interference models," in IEEE Radar Conference, 2019, in press. 
[28] S. H. Dokhanchi, B. S. Mysore, K. V. Mishra, and B. Ottersten, "A mmWave automotive joint radar-communications system," IEEE Transactions on Aerospace and Electronic Systems, vol. 55, pp. 12411260, 2019.

[29] G. Duggal, S. Vishwakarma, K. V. Mishra, and S. S. Ram, "Dopplerresilient 802.11ad-based ultra-short range automotive radar," arXiv preprint arXiv:1902.01306, 2019.

[30] M. Palola, M. Matinmikko, J. Prokkola, M. Mustonen, M. Heikkilä, T. Kippola, S. Yrjölä, V. Hartikainen, L. Tudose, A. Kivinen et al., "Live field trial of licensed shared access (LSA) concept using LTE network in $2.3 \mathrm{GHz}$ band," in IEEE International Symposium on Dynamic Spectrum Access Networks, 2014, pp. 38-47.

[31] P. Liu, Y. Liu, T. Huang, Y. Lu, and X. Wang, "Cognitive radar using reinforcement learning in automotive applications," arXiv preprint
arXiv:1904.10739, 2019.

[32] K. V. Mishra, A. Martone, and A. I. Zaghloul, "Power allocation games for overlaid radar and communications," in URSI Asia-Pacific Radio Science Conference (AP-RASC), 2019, pp. 1-4.

[33] A. M. Elbir and K. V. Mishra, "Joint antenna selection and hybrid beamformer design using unquantized and quantized deep learning networks," arXiv preprint arXiv: 1905.03107, 2019.

[34] P. Stoica and A. Nehorai, "MUSIC, maximum likelihood, and CramérRao bound: Further results and comparisons," IEEE Transactions on Acoustics, Speech, and Signal Processing, vol. 38, no. 12, pp. 21402150, 1990.

[35] R. Schmidt, "Multiple emitter location and signal parameter estimation," IEEE Transactions on Antennas and Propagation, vol. 34, no. 3, pp. 276-280, 1986. 